\title{
Clinical and Lab-Assessed Antibiotic Resistance Pattern of Uropathogens among Women with Acute Uncomplicated Cystitis
}

\author{
Ahmed M. Abdelkhalik', Mohamed M. Agha ${ }^{1}$, Ahmed M. Zaki ${ }^{1}$, Ahmed Tahoun ${ }^{2}$ \\ ${ }^{1}$ Urology and ${ }^{2}$ Clinical Pathology Departments, Faculty of Medicine, Al-Azhar University, Cairo, \\ Egypt \\ Corresponding author: Mohamed M. Agha, Mobile: 01091224033; \\ Email: Aghaurology@yahoo.com
}

\begin{abstract}
Background: acute uncomplicated cystitis (AUC) in females is among the most frequent infections in community. Treatment of AUC in Egypt usually based on empirical therapy.

Objectives: To determine the most common uropathogens and their pattern of resistance to commonly used antimicrobials aiming at finding a proper antibiotic which can be used as effective empirical therapy in AUC.

Patients and Methods: Observational, hospital based, cross sectional study was performed. Three hundred and twelve female patients were recruited for the study with positive urine culture and clinical symptoms correlate with AUC during the period from December 2017 to August 2018.

Results: Eight organisms were isolated. E. coli was the most common uropathogen and was isolated from 231 cases $(74 \%)$. The next most common was Pseudomonas which was detected in 41 cases (13.1\%). Other organisms include MRSA (6.1\%), Klebsiella (2.2\%), Enterococcus spp. (1.9\%), proteus $(1.3 \%)$, Acinetobacter and Staph. aureus $(0.6 \%)$ each. Isolated uropathogens in this study were least resistant to Nitrofurantoin (47.1\%). The highest resistance rate was to TMP/SMZ (87.8\%). Resistance pattern to quinolones were $64.1 \%, 67 \%$ and $60.6 \%$ to Ciprofloxacin, Norfloxacinand and Levofloxacin respectively.

Conclusion: The most common cause of community acquired AUC was E. coli. Nitrofurantoin can be used as a first line empirical therapy for AUC as it showed the least resistance pattern (47.1\%). Antimicrobial resistance is a crucial issue which evolves continuously and thus more studies must be carried out in different regions and constanttime intervals.
\end{abstract}

Key words: Urinary tract infection, Acute uncomplicated cystitis, Antibiotic Resistance Pattern.

\section{Introduction}

Urinary tract infections are one of the most common infections in women ${ }^{(1)}$. Acute uncomplicated cystitis refers to a bladder infection that occurs in women who have normal structure and function of the genitourinary $\operatorname{tract}^{(2)} . \quad 10 \%$ of women experience at least one episode of acute uncomplicated urinary infection in a year, and $60 \%$ have at least one episode during their lifetime ${ }^{(3)}$. The peak incidence of infection occurs in young, sexually active women aged 18 to 24 years ${ }^{(4)}$. E. coli accounts for $85 \%$ of community acquired and $50 \%$ of hospital acquired urinary tract infections. Gram-positive Enterococcus faecalis and Staphylococcus saprophyticus are causative agents for the remainder of community acquired infections ${ }^{(5)}$. Development of acute uncomplicated urinary infection follows colonization of the vagina and the periurethral area by uropathogenic E. $\operatorname{coli}^{(6)}$. Acute uncomplicated urinary infection has a characteristic clinical presentation. New onset frequency, dysuria, and urgency, together with the absence of vaginal discharge or pain, has a positive predictive value of $90 \%$ for acute cystitis $^{(7)}$. Diagnosis may be confirmed by urine analysis, urine culture and sensitivity test and may be pelvi-abdominal ultrasonography to exclude complicated cases. Significant bacteriuria refers to bacterial colonization $\geq 10^{5} \mathrm{cfu} / \mathrm{ml}^{(8)}$. The World Health Organization has named antibiotic resistance as one of the three most important public health threats of the 21 st century ${ }^{(9)}$. It was estimated that at least 23,000 people die annually in the USA as a result of an infection with an antibiotic-resistant organism $^{(10)}$. Antibiotic resistance is estimated to cause around 300 million premature deaths by $2050^{(11)}$. Knowledge of the antimicrobial resistance patterns of common uropathogens is essential to provide an effective empirical therapy for AUC. 


\section{Patients and Methods Patients:}

The study was performed in Urology outpatient clinics of Al-Azhar University hospitals; 312 adult female patients presented with acute uncomplicated cystitis were recruited from December 2017 to August 2018. Patients were presented by one or more of the following symptoms; dysuria, frequency, urgency, incontinence, hematuria, suprapubic pain or loin pain. Patients with the following criteria were excluded; Recurrent UTI (three or more episodes of UTI in the past year), pregnancy, diabetes mellitus and other immunecompromised conditions, urinary tract anomalies (anatomical or functional disorders), urinary catheterization, current antibiotic use or history of neurological disorders.

\section{Urine sample collection and processing:}

After detailed medical and surgical history and once diagnosis of AUC was established, patients were informed and consented about the study and urine samples were collected. Every patient was carefully instructed about proper cleaning of external genitalia and collection of clean catch mid-stream urine sample. The urine samples were taken to the Microbiology laboratory, Faculty of Medicine, Al-Azhar University within two hours at most where they were examined microscopically for pus cells and inoculated immediately on blood agar and MacConkey's agar plates. Colony counting, isolation and identification of the urinary pathogens were done according to standard bacteriological techniques. Culture result with a bacterial growth of $\geq 10^{5} \mathrm{cfu} / \mathrm{ml}$ was considered as significant bacteruria ${ }^{(12)}$.

\section{Antimicrobial susceptibility testing:}

Antimicrobial susceptibility testing was performed using the disc diffusion method as described by the National Committee for
Clinical Laboratory Standards (13). Antimicrobial agents discs tested and reported include, Norfloxacin (NOR10 $\mu \mathrm{g}$ ), Ciprofloxacin (CIP10 $\mu$ g), AmpicillinSulbactam (SAM 10/10 $\mu$ g), Amoxicillin/Clavulanic acid (AMC 20/10 $\mu \mathrm{g}$ ), Gentamicin(CN10 $\mu \mathrm{g})$, Cefotaxime (CTX30 $\mu \mathrm{g})$, Ceftriaxone(CRO30 $\mu \mathrm{g})$, Trimethoprim/Sulfamthoxazole (SXT $1.25 / 23.75 \mu \mathrm{g}$ ) and Nitrofurantoin (F $300 \mu \mathrm{g}$ ). Isolated organisms were tested for susceptibility on the following antimicrobials, Nitrofurantoin, TrimethoprimSulfamethoxazole (TMP-SMZ), Ciprofloxacin, Norfloxacin, Levofloxacin, Ceftriaxone, Cefotaxime, Amoxicillin/Clavulanic acid, Ampicillin/sulbactam and Gentamicin.

\section{Antibiotic prescribed:}

After collection of urine samples, patients were treated empirically with ciprofloxacin. TMP/SMZ was prescribed to cases (46 cases) who had history of ciprofloxacin hypersensitivity or others who can't tolerate ciprofloxacin due to GIT upsets. Patients were followed after one week for detection of clinical response.

\section{Results}

Out of 379 adult female patients recruited for the study, 312 patients were eligible for the study with a positive urine culture and clinical features compatible with AUC. Sixteen patients $(4.2 \%)$ were discovered by completed assessment to have anatomic or functional abnormalities, 28 patients $(7.3 \%)$ did not come for follow up and 23 patients $(6 \%)$ their culture and sensitivity tests revealed no bacterial growth. The age of selected cases was classified into three categories. Most of cases, 218 patients $(69.9 \%)$, were below 40 years old, while 69 patients $(22.1 \%)$ were found in the zone of 40 to 60 years old. Least cases, 25 patients $(8 \%)$, were more than 60 years old.

Table (1): Age distribution in studied cases

\begin{tabular}{|l|c|c|c|}
\hline Age groups & Frequency & Percent & Cumulative Percent \\
\hline Less Than 40 years & 218 & 69.9 & 69.9 \\
\hline 40-60 years & 69 & 22.1 & 92 \\
\hline More than 60 years & 25 & 8 & 100 \\
\hline Total & 312 & 100 & \\
\hline
\end{tabular}

In studied cases, 233 cases (74.7\%) were married while 79 (25.3\%) were not. 
Clinical and Lab-Assessed Antibiotic Resistance Pattern of Uropathogens among Women...

Table (2): Marital status of studied cases

\begin{tabular}{|l|c|c|c|c|}
\hline \multicolumn{5}{|c|}{ Marital status of selected cases } \\
\hline Marital status & Frequency & Percent & Valid Percent & Cumulative Percent \\
\hline Not Married & 79 & 25.3 & 25.3 & 25.3 \\
\hline Married & 233 & 74.7 & 74.7 & 100.0 \\
\hline Total & 312 & 100.0 & 100.0 & \\
\hline
\end{tabular}

By urine culture and sensitivity tests, eight species of bacteria were detected. The most common organism was E. coli which was detected in 231 cases (74\%). The next most common was Pseudomonas which was detected in 41 cases (13.1\%). Other organisms include; MRSA (6.1\%), klebsiella $(2.2 \%)$, enterococcus $(1.9 \%)$, proteus (1.3), acinetobacter and Staph. aureus $(0.6 \%)$ each.

Table (3): Bacterial species detected by culture and sensitivity tests of urine samples of studied cases.

\begin{tabular}{|l|c|c|c|c|}
\hline \multicolumn{5}{|c|}{ Organisms } \\
\hline \multicolumn{1}{|c|}{ Organism } & Frequency & Percent & Valid Percent & Cumulative Percent \\
\hline E. coli & 231 & 74.0 & 74.0 & 74.7 \\
\hline Enterococcus & 6 & 1.9 & 1.9 & 76.6 \\
\hline Klebsiella & 7 & 2.2 & 2.2 & 78.8 \\
\hline MRSA & 19 & 6.1 & 6.1 & 84.9 \\
\hline Proteus & 4 & 1.3 & 1.3 & 86.2 \\
\hline Pseudomonas & 41 & 13.1 & 13.1 & 99.4 \\
\hline Staph. aureus & 2 & 0.6 & 0.6 & 100.0 \\
\hline Total & 312 & 100.0 & 100.0 & \\
\hline
\end{tabular}

The most common uropathogen in middle aged group (20-40 years old) was E. coli. Out of 202 cases at middle age, $144 \mathrm{E}$. coli isolates were detected (71.2\%). Acinetobacter, klebsiella, MRSA, proteus and Staph. aureus were not detected at all below age of 20 years. Enterococcus and Staph. aureus were also absent above age of 40 years.

Table (4): Frequency of prevalent organisms in relation to patient's age

\begin{tabular}{|c|c|c|c|c|c|c|c|c|c|c|}
\hline \multicolumn{2}{|c|}{ Organism } & \multirow{2}{*}{ 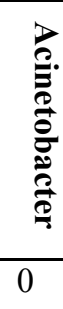 } & \multirow{2}{*}{$\begin{array}{l}\stackrel{\Gamma}{9} \\
\stackrel{8}{=} \\
14\end{array}$} & \multirow{2}{*}{ 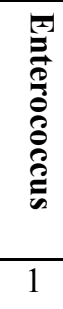 } & \multirow{2}{*}{$\begin{array}{l}\frac{\pi}{\alpha} \\
\frac{a}{0} \\
\frac{0}{0} \\
\frac{0}{20} \\
0\end{array}$} & \multirow{2}{*}{ 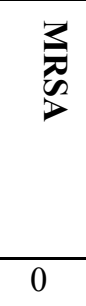 } & \multirow{2}{*}{$\begin{array}{l}\overline{0} \\
\overline{0} \\
\overline{0} \\
\overline{0}\end{array}$} & \multirow{2}{*}{ 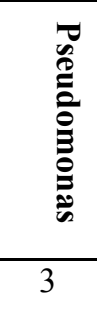 } & \multirow{2}{*}{ 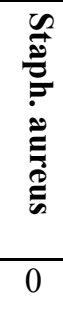 } & \multirow{2}{*}{ 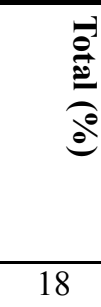 } \\
\hline \multirow{2}{*}{$<\mathbf{2 0}$} & Number & & & & & & & & & \\
\hline & Percentage & 0.0 & 4.5 & 0.3 & 0.0 & 0.0 & 0.0 & 1.0 & 0.0 & 5.8 \\
\hline \multirow{2}{*}{$20-40$} & Number & 1 & 144 & 5 & 4 & 13 & 3 & 30 & 2 & 202 \\
\hline & Percentage & 0.3 & 46.2 & 1.6 & 1.3 & 4.2 & 1.0 & 9.6 & 0.6 & 64.7 \\
\hline \multirow[b]{2}{*}{$>40$} & Number & 1 & 73 & 0 & 3 & 6 & 1 & 8 & 0 & 92 \\
\hline & Percentage & 0.3 & 23.4 & 0.0 & 1.0 & 1.9 & 0.3 & 2.6 & 0.0 & 29.5 \\
\hline \multirow{2}{*}{ Total } & Number & 2 & 231 & 6 & 7 & 19 & 4 & 41 & 2 & 312 \\
\hline & Percentage & 0.6 & 74.0 & 1.9 & 2.2 & 6.1 & 1.3 & 13.1 & 0.6 & 100.0 \\
\hline
\end{tabular}

Antibiotics sensitivity was tested against 10 antibiotics which are the commonly prescribed antibiotics in our society. These antibiotics are Nitrofurantoin, Ciprofloxacin, Norfloxacin, Levofloxacin, Trimethoprium/ sulfamethoxazole (TMP/SMZ), Ceftriaxone, Cefotaxime, Amoxicillin/ Clavulanic acid, Ampicillin/Sulbactam and Gentamicin. 
Nitrofurantoin showed the least resistance pattern. Out of 312 cases, 165 cases were sensitive to Nitrofurantoin $(52.9 \%)$ while 147 cases were resistant to it (47.1\%).

Table (5): Sensitivity and resistance of Uropathogens to nitrofurantoin

\begin{tabular}{|c|c|c|c|c|}
\hline Nitrofurantoin & Frequency & Percent & $\begin{array}{c}\text { Valid } \\
\text { Percent }\end{array}$ & $\begin{array}{c}\text { Cumulative } \\
\text { Percent }\end{array}$ \\
\hline S & 165 & 52.9 & 52.9 & 52.9 \\
\hline R & 147 & 47.1 & 47.1 & 100.0 \\
\hline Total & 312 & 100.0 & 100.0 & \\
\hline
\end{tabular}

TMP/SMZ showed the least sensitivity pattern in selected cases. In 312 isolates, 38 cases $(12.2 \%)$ were sensitive to TMP/SMZ while 274 cases $(87.8 \%)$ were resistant.

Table (6): Sensitivity and resistance of TMP/SMZ in selected cases

\begin{tabular}{|c|c|c|c|c|}
\hline TMP/SMZ & Frequency & Percent & Valid Percent & $\begin{array}{c}\text { Cumulative } \\
\text { Percent }\end{array}$ \\
\hline S & 38 & 12.2 & 12.2 & 12.2 \\
\hline R & 274 & 87.8 & 87.8 & 100.0 \\
\hline Total & 312 & 100.0 & 100.0 & \\
\hline
\end{tabular}

Resistance and sensitivity pattern of antimicrobials to different Quinolones were quite similar. Ciprofloxacin showed sensitivity in 112 cases (35.9\%) and resistance in 200 cases (64.1\%). Norfloxacin showed sensitivity in 103 cases (33\%) and resistance in 209 cases (67\%). Levofloxacin showed sensitivity in 123 cases $(39.4 \%)$ and resistance in 189 cases $(60.6 \%)$.

Table (7): Sensitivity and resistance of uropathogs to ciprofloxacin

\begin{tabular}{|c|c|c|c|c|}
\hline Ciprofloxacin & Frequency & Percent & Valid Percent & $\begin{array}{c}\text { Cumulative } \\
\text { Percent }\end{array}$ \\
\hline S & 112 & 35.9 & 35.9 & 35.9 \\
\hline R & 200 & 64.1 & 64.1 & 100.0 \\
\hline Total & 312 & 100.0 & 100.0 & \\
\hline
\end{tabular}

Table (8): Sensitivity and resistance of uropathogens to norfloxacin

\begin{tabular}{|c|c|c|c|c|}
\hline Norfloxacin & Frequency & Percent & Valid Percent & Cumulative Percent \\
\hline S & 103 & 33.0 & 33.0 & 33.0 \\
\hline R & 209 & 67.0 & 67.0 & 100.0 \\
\hline Total & 312 & 100.0 & 100.0 & \\
\hline
\end{tabular}

Table (9): Sensitivity and resistance of uropathogens to Levofloxacin

\begin{tabular}{|c|c|c|c|c|}
\hline Levofloxacin & Frequency & Percent & Valid Percent & Cumulative Percent \\
\hline S & 123 & 39.4 & 39.4 & 39.4 \\
\hline R & 189 & 60.6 & 60.6 & 100.0 \\
\hline Total & 312 & 100.0 & 100.0 & \\
\hline
\end{tabular}

E. coli strains showed the least rate of resistance to Nitrofurantoin and the highest rate of resistance to TMP/SMZ. No organism were absolutely resistant to Nitrofurantoin while Klebsiella, Enterococcus, Proteus, Staph. aureus and Acinetobacter were absolutely resistant to TMP/SMZ. Table (10): Overall resistance (\%) of uropathogenes to antimicrobials in selected cases with acute uncomplicated cystitis 
Clinical and Lab-Assessed Antibiotic Resistance Pattern of Uropathogens among Women...

\begin{tabular}{|c|c|c|c|c|c|c|c|c|c|c|}
\hline resistance & 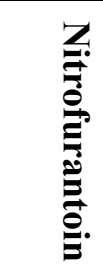 & 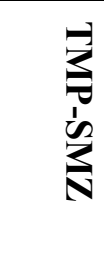 & 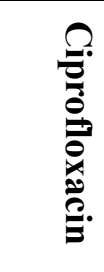 & 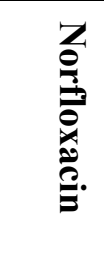 & 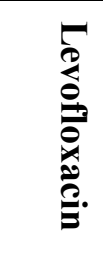 & 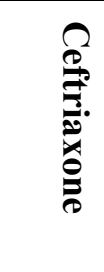 & 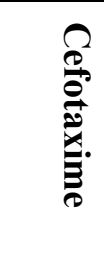 & $\begin{array}{l}\mathrm{Z} \\
\mathbf{3} \\
\frac{0}{2} \\
\frac{2}{20} \\
\frac{0}{2}\end{array}$ & 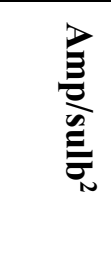 & 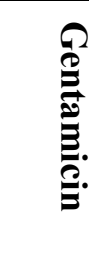 \\
\hline E.coli & 48.1 & 87.4 & 65.4 & 68.4 & 62.3 & 74.9 & 64.9 & 68.4 & 81.4 & 77.9 \\
\hline Pseudomonas & 36.6 & 85.4 & 65.9 & 43.9 & 61 & 63.4 & 61 & 63.4 & 73.2 & 75.6 \\
\hline MRSA & 57.9 & 84.2 & 52.6 & 57.9 & 31.6 & 57.9 & 57.9 & 73.7 & 94.7 & 89.5 \\
\hline Klebsiella & 42.9 & 100 & 28.6 & 71.4 & 28.6 & 57.1 & 57.1 & 42.9 & 100 & 85.7 \\
\hline Enterococcus & 33.3 & 100 & 50 & 83.3 & 83.3 & 83.3 & 83.3 & 50 & 66.7 & 100 \\
\hline Proteus & 75 & 100 & 100 & 100 & 100 & 75 & 75 & 50 & 25 & 75 \\
\hline Staph aureus & 50 & 100 & 50 & 50 & 50 & 50 & 50 & 50 & 100 & 50 \\
\hline Actinetobacter & 50 & 100 & 100 & 100 & 100 & 100 & 100 & 50 & 50 & 50 \\
\hline
\end{tabular}

*(1) Amoxicillin-clavulaniccid, * (2) Ampicillin-sulbactam

MDR referred to uropathogens which were resistant to more than three groups of antimicrobials. Overall MDR were detected in 244 cases (78.2\%). Out of $231 \mathrm{E}$. coli isolated strains, MDR were detected in 185 cases $(80 \%)$ of all E. coli isolates which represent $59.3 \%$ of total MDR.

Table (11): Multidrug resistanc of different uropathogens for the tested antimicrobials

\begin{tabular}{|c|c|c|c|c|c|c|}
\hline \multicolumn{2}{|c|}{ Organisms } & \multirow{2}{*}{ 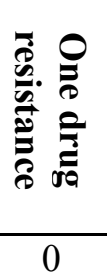 } & \multirow{2}{*}{ 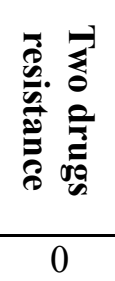 } & \multirow{2}{*}{ 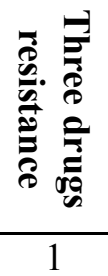 } & \multirow{2}{*}{ 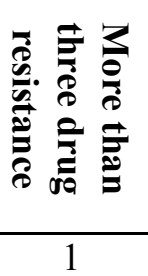 } & \multirow{2}{*}{$\frac{\vec{\theta}}{\stackrel{\overrightarrow{0}}{=}}$} \\
\hline & Count & & & & & \\
\hline Acinetobacter & percent & 0.00 & 0.00 & 0.30 & 0.30 & 0.60 \\
\hline \multirow{2}{*}{ E. coli } & Count & 0 & 11 & 35 & 185 & 231 \\
\hline & percent & 0.00 & 3.50 & 11.20 & 59.30 & 74.00 \\
\hline \multirow{2}{*}{ Enterococcus } & Count & 0 & 0 & 0 & 6 & 6 \\
\hline & percent & 0.00 & 0.00 & 0.00 & 1.90 & 1.90 \\
\hline \multirow{2}{*}{ Klebsiella } & Count & 0 & 0 & 0 & 7 & 7 \\
\hline & percent & 0.00 & 0.00 & 0.00 & 2.20 & 2.20 \\
\hline \multirow{2}{*}{ MRSA } & Count & 0 & 2 & 5 & 12 & 19 \\
\hline & percent & 0.00 & 0.60 & 1.60 & 3.80 & 6.0 \\
\hline \multirow{2}{*}{ Proteus } & Count & 0 & 1 & 0 & 3 & 4 \\
\hline & percent & 0.00 & 0.30 & 0.00 & 1.00 & 1.30 \\
\hline \multirow{2}{*}{ Pseudomonas } & Count & 0 & 4 & 7 & 30 & 41 \\
\hline & percent & 0.00 & 1.30 & 2.20 & 9.60 & 13.10 \\
\hline \multirow{2}{*}{ Staph. aureus } & Count & 1 & 1 & 0 & 0 & 2 \\
\hline & percent & 0.30 & 0.30 & 0.00 & 0.00 & 0.6 \\
\hline \multirow{2}{*}{ Total } & Count & 1 & 19 & 48 & 244 & 312 \\
\hline & percent & 0.30 & 6.10 & 15.40 & 78.20 & 100.00 \\
\hline
\end{tabular}

Ciprofloxacin was prescribed empirically in 266 cases $(85.3 \%)$ while TMP/SMX was prescribed in 46 cases $(14.7 \%)$. 
Table (12): Empirical antibiotics prescribed for studied cases

\begin{tabular}{|l|c|c|c|c|}
\hline \multicolumn{7}{|c|}{ Antibiotics prescribed } \\
\hline \multicolumn{1}{|c|}{ Antibiotic } & Frequency & Percent & Valid Percent & Cumulative Percent \\
\hline Ciprofloxacin & 266 & 85.3 & 85.3 & 85.3 \\
\hline TMP-SMZ & 46 & 14.7 & 14.7 & 100.0 \\
\hline Total & 312 & 100.0 & 100.0 & \\
\hline
\end{tabular}

The clinical response of cases was determined after 7 days course of antibiotic. 279 cases improved (89.4\%) while 33 cases didn't improve (10.6\%).

Table (13): Clinical response of selected cases to empirical antibiotics prescribed

\begin{tabular}{|c|c|c|c|c|}
\hline \multicolumn{5}{|c|}{ Clinical response } \\
\hline Response & Frequency & Percent & $\begin{array}{c}\text { Valid } \\
\text { Percent }\end{array}$ & $\begin{array}{c}\text { Cumulative } \\
\text { Percent }\end{array}$ \\
\hline Improved & 279 & $89.4 \%$ & $89.4 \%$ & 89.4 \\
\hline Not improved & 33 & $10.6 \%$ & $10.6 \%$ & 100.0 \\
\hline Total & 312 & $100.0 \%$ & $100.0 \%$ & \\
\hline
\end{tabular}

In cases treated empirically with ciprofloxacin, 237 cases improved clinically (89.1\%) while 29 cases didn't improve (10.9\%). In cases empirically treated with TMP/SMZ, 42 cases improved clinically (91.3\%) while 4 cases didn't improve (8.7\%).

Table (14): Clinical response of selected cases to ciprofloxacin and TMP/SMZ

\begin{tabular}{|c|c|c|c|c|c|}
\hline \multirow{2}{*}{ Antibiotic } & Response & Frequency & Percent & $\begin{array}{c}\text { Valid } \\
\text { Percent }\end{array}$ & $\begin{array}{c}\text { Cumulative } \\
\text { Percent }\end{array}$ \\
\hline \multirow{3}{*}{ Ciprofloxacin } & Improved & 237 & 89.1 & 89.1 & 89.1 \\
\cline { 2 - 6 } & Not improved & 29 & 10.9 & 10.9 & 100.0 \\
\cline { 2 - 6 } & Total & 266 & 100.0 & 100.0 & \\
\hline \multirow{3}{*}{ TMP-SMZ } & Improved & 42 & 91.3 & 91.3 & 91.3 \\
\cline { 2 - 6 } & Not improved & 4 & 8.7 & 8.7 & 100.0 \\
\cline { 2 - 6 } & Total & 46 & 100.0 & 100.0 & \\
\hline
\end{tabular}

In this study, 23 cases were excluded from the study after culture and sensitivity tests revealed no bacterial growth. Out of these cases, 21 cases received ciprofloxacin, while 2 cases received TMP/SMZ. On follow up, 19 cases $(82.6 \%)$ improved while 4 cases $(17.4 \%)$ didn't improve. Of ciprofloxacin prescribed cases 18 cases $(85.7 \%)$ improved while 3 cases (14.3\%) didn't. Of TMP/SMZ prescribed cases 1 case $(50 \%)$ improved while 1 case $(50 \%)$ didn't.

\section{Discussion}

This study included 312 patients with positive cultures, including total of eight species, three gram positive species; namely MRSA in 19 cases $(6.1 \%)$, Enterococcus in 6 cases $(1.9 \%)$, Staphylococcus aureus in 2 cases $(0.6 \%)$ and five gram negative species; namely E coli presenting the most predominant species in 231 cases(74\%), pseudomonas in 41cases $(13.1 \%)$, klebsiella in 7 cases $(2.2 \%)$, proteus in 4 cases $(1.3 \%)$, and Acinetobacter in 2 cases $(0.6 \%)$.

Our results showed that E. coli was the most common bacterium that caused urinary tract infections (74\%), and this result agrees with previous studies both in Egypt and other countries $^{(14,15,16)}$. In the current study, the most frequent uropathogens included in acute uncomplicated cystitis were E. coli (74\%), Pseudomonas (13.1\%), MRSA (6.1\%), Klebseilla (2.2\%) and Enterococcus (1.9\%). In previous study done in Egypt, the prevalence of uropathogens was not the same. E. coli was found in $61.76 \%$, Klebseilla $\quad 12.5 \%$, Enterococcus $\quad 7.35 \%$, Staphylococcus aureus $6.61 \%$ and Pseudomonas $3.76 \%{ }^{(14)}$.

Although the demographic difference doesn't affect the fact that E. coli is the most 
predominant organism causing acute uncomplicated cystitis, there were significant demographic changes regarding uropathogens other than E.coli. In a similar study performed at Saudia Arabia, E. coli represents 53\% of the isolates while Klebsiella was $15 \%$, Pseudomonas $12 \%$, MRSA 3\%, Enterococci 3\%, Enterobacter $3 \%$ and Proteus $2 \%{ }^{(15)}$. In an Indian study, E. coli represents $67.66 \%$ of isolates. The next most common uropathogen was Klebsiella pneumonia (14\%) followed by Proteus sp. (6\%), Pseudomonas (8\%), Enterococcus sp. (2\%), and Streptococcus agalactiae $(1 \%)^{(16)}$. In an Italian study, E. coli represents $85.3 \%$ of isolates, followed by Klebsiella pneumonia (6\%), Staphylococcus saprophyticus (3.2\%), Proteus Mirabilis (2.3\%), Enterococcus faecalis $(1.7 \%)^{(17)}$. In a similar study performed at South Korea, E. coli represents $71.1 \%$ of isolates. The second most common uropathogen was Enterococci (13.0\%) followed by Klebsiella spp. (3.7\%) Enterobacter spp. (3.0\%) Citrobacter spp. $(3.0 \%)$ Proteus spp. $(1.0 \%)^{(18)}$. In a Brazilian study, E. coli represents $64.7 \%$ of isolates. The second most common uropathogen was Klebsiella sp. (14.2\%), Enterobacter sp. (7.1\%) followed by Proteus sp. (4.8\%), coagulasenegative staphylococcus (4.3\%), Citrobacter sp. (2.4\%), Enterococcus sp. (1.2\%), Staph. aureus $(0.8 \%)$ and Pseudomonas sp. $(0.5 \%)^{(19)}$. In a study performed in USA, E. coli represents $68 \%$ of isolates. The second most common uropathogen was Staphylococcus saprophyticus $(8 \%)$, followed by Group B streptococci (7\%), Proteus spp. (6\%), Klebsiella spp. (4\%), Enterococcus $(3 \%)$, and other organisms $(4 \%)^{(20)}$.

This study was concerned about detecting pattern of resistance of uropathogenes to antimicrobials which are commonly used in our society. These antimicrobials include: Nitrofurantoin, Trimethoprim/ sulfamethoxazole (TMP-SMZ), Ciprofloxacin, Levofloxacin, Norfloxacin, Ceftriaxone, Cefotaxime, Amoxicillin/Clavulanic acid, Ampicillin/ Sulbactum and Gentamicin.

In this study, resiatance to Nitrofurantoin was $47.1 \%$, TMP-SMZ 87.8\%, Ciprofloxacin $64.1 \%$, Levofloxacin $60.6 \%$, Norfloxacin $67 \%$, ceftriaxone $72.1 \%$, Cefotaxime $64.4 \%$, Amoxicillin/Clavulanic acid 66.7\%, Ampicillin/Sulbactum 79.8\% and Gentamicin $78.5 \%$. These data indicate that among common antibiotics tested in our study, only nitrofurantoin had less than $50 \%$ resistance $(47.1 \%)$ which supports its recommendation as an effective empiric therapy for AUC in our hospitalscommunity area. In an Egyptian study performed in 2009, resistance of E. coli was $17.4 \%$ to Nitrofurantoin, $60 \%$ to TMP-SMZ, $47.8 \%$ for Ciprofloxacin and Norfloxacin, $40.7 \%$ for Ceftriaxone, $39.1 \%$ for Cefotaxime, $68.1 \%$ for Amoxicillin/Clavulanic acid, $100 \%$ for Ampicillin/Sulbactam and $34.8 \%$ for Gentamicin ${ }^{(14)}$. In a study performed in Saudia Arabia, resistnce to Nitrofurantoin was $22.4 \%$, TMP-SMZ 59.5\%, Ciprofloxacin 32.4\%, Norfloxacin $\quad 46 \%$, Ceftriaxone $32.2 \%$, Amoxicillin/Clavulanic acid $51.6 \%$ and Gentamicin $32.3 \%{ }^{(15)}$. In an Indian study, the pattern of resistance of E.coli was quite similar. Resistance of E. coli strains to Nitrofurantoin was 36.1\%, TMP-SMZ100\%, Amoxicillin/Clavulanic acid $85 \%$, Aminoglycosides $67 \%$, Fluoroquinolones $75 \%$ and Ceftriaxone $65 \%{ }^{(16)}$. In a Brazillin study, the pattern of resistance was quite different where resistance to Nitrofurantoin was $15.7 \%$, TMP-SMZ $39.8 \%$, Cprofloxacin $10.2 \%$, Norfloxacin $7.5 \%$, Levofloxacin $18.2 \%$, Ceftriaxone 4.9\%, Amoxicillin/Clavulanic acid $17.4 \%$ and Gentamicin $3.5 \%{ }^{(19)}$. In a similar study performed in Italy, resistant pattern was also different where Nitrofurantoin was 9.9\%, TMPSMX 30.2\%, Ciprofloxacin 15\%, levofloxacin $15 \%$ and amoxicillin/Clavulanic acid $11.7 \%{ }^{(17)}$. In a similar study performed in South Korea, resistance to TMP-SMZ was $26.1 \%$, Ciprofloxacin 21.1\%, Ampicillin/Sulbactam $47.7 \%$ and Gentamicin $19.5 \%{ }^{(18)}$.

In this study, multi-drug resistant E. coli strains represent $59.3 \%$. In an Indian study, multi-drug resistant E. coli strains represent $65 \%{ }^{(16)}$.

Out of 312 cases, Ciprofloxacin were prescribed empirically in 266 cases $(85.3 \%)$ while TMP/SMZ was prescribed in 46 cases (14.7\%). Out of 312 cases, 279 cases $(89.4 \%)$ improved with empirical therapy while 33 cases (10.6\%) didn't improve. Out of 266 cases ciprofloxacin was prescribed for, 237 cases improved clinically (89.1\%) while 29 cases didn't improve (10.9\%). Out of 46 cases TMP/SMZ was described for, 42 cases improved clinically (91.3\%) while 4 cases didn't improve (8.7\%).

\section{Conclusion}

This study showed that E. coli is the most common pathogen involved in AUC. Antibiotic resistance is a worldwide problem threatening our ability to treat infections and 
thus increase rate of treatment failure which in turn increases mortality, morbidity and economic costs. Isolated uropathogens in this study were least resistant to Nitrofurantoin $(47.1 \%)$. The highest resistance rate was to TMP/SMZ (87.8\%). Resistance pattern to quinolones were $64.1 \%, 67 \%$ and $60.6 \%$ to ciprofloxacin, norfloxacin and levofloxacin respectively.

\section{Recommendations}

From our study, we recommend that Nitrofurantoin is suitable to be the first line of empirical therapy for treating AUC as it shows the least resistance pattern among studied antibiotics. We also recommend that this study should be followed by similar community studies at different regions and constant intervals to detect pattern of uropathogens resistance to commonly used antimicrobials and whether it evolve or not. This will help clinicians to provide safe and effective empirical therapies with minimal therapeutic failures.

\section{References}

1. Foxman B (2002): Epidemiology of urinary tract infections: incidence, morbidity and economic costs. Am J Med., 113 (1A):5-13.

2. Hooton TM (2003): The current management strategies for communityacquired urinary tract infection. Infect Dis Clin North Am., 17:303-22.

3. Foxman B, Barlow R, D'Arcy $\mathrm{H}$ et al. (2000): Urinary tract infection: selfreported incidence and associated costs. Ann Epidemiol., 10:509-15.

4. Fihn SD (2003): Acute uncomplicated urinary tract infection in women. $\mathrm{N}$ Engl $\mathrm{J}$ Med., 349:259-66.

5. Brooks HJ, Benseman BA, Peck J et al. (1981): Correlation between uropathogenic properties of Escherichia coli from urinary tract infections and the antibody-coated bacteria test and comparison with faecal strains, J Hyg (Lond), 87(1): 53-61.

6. Gupta K, Hillier SL, Hooton TM et al. (2000): Effects of contraceptive method on the vaginal microbial flora: a prospective evaluation. J Infect Dis., 181: 595-601.

7. Bent S, Nallamothu BK, Simel DL et al. (2002): Does this women have an acute uncomplicated urinary tract infection? JAMA., 287:2701-10.
8. Mulvey MA, Lopez-Boado YS, Wilson CL et al. (1998): Induction and evasion of host defenses by type 1-piliated uropathogenic Escherichia coli. Science, 282:1494-7.

9. Antimicrobial resistance (2014): global report on surveillance 2014. World HealthOrganization. http:// www. who. int/ drugresistance/ documents/ surveillancereport/ en/

10. Centers for Disease Control and
Prevention (2013): Antibiotic Resistance, Threats in the United States. Downloaded from http:// www. cdc. gov/ drugresistance/ threat report2013/index.html.

11. O'Neill J (2014): Antimicrobial resistance: tackling a crisis for the health and wealth of nations. Rev. Antimicrob. Resist., 20:1-6.

12. Cruickshank R, Duguid JP, Marmion BP (1996): Tests for identification of bacteria. In: Medical Microbiology, Vol 2, 12th ELBS ed. London: Churchill Livingstone, pp. 170- 189.

13. Reller LB, Weinstein M, Jorgensen JH, Ferraro MJ (2009): Antimicrobial susceptibility testing: a review of general principles and contemporary practices. Clinical infectious diseases, 49(11):174955.

14. Labah E, Afifi I, Ahmed L (2009): Community-Acquired Urinary Tract Infections in Tanta, Egypt: Aetiology and Antibiotic Resistance Pattern. Egyptian J. of Med. Microbiology, 18 (4): 179-189.

15. Tarek AS and Mohamed $H$ (2011): Uropathogens causing urinary tract infections in females and their susceptibility to antibiotics. Uro Today Int J. , 4:1944-5784.

16. Sharma N, Gupta A, Walia G, Bakhshi R (2016): Pattern of Antimicrobial Resistance of Escherichia coli Isolates from Urinary Tract Infection Patients: A Three Year Retrospective Study. J App Pharm Sci., 6(01):062-5.

17. Fadda G, Nicoletti G, Schito GC, Tempera G (2005): Antimicrobial susceptibility patterns of contemporary pathogens from uncomplicated urinary tract infections isolated in a multicenter Italian survey: possible impact on guidelines. J Chemother., 17(3):251-7.

18. Kim ME, Ha US, Cho YH (2008): 
Clinical and Lab-Assessed Antibiotic Resistance Pattern of Uropathogens among Women...

Prevalence of antimicrobial resistance among uropathogens causing acute uncomplicated cystitis in female outpatients in South Korea: a multicentre study in 2006. International journal of antimicrobial agents, 31:15-8.

19. Araújo SM, Mourão TC, Oliveira JL, Melo IF, Araújo CA, Araújo NA, Melo MC, Araújo SR, Daher EF (2011): Antimicrobial resistance of uropathogens in women with acute uncomplicated cystitis from primary care settings. Int Urol Nephrol., 43(2):461-6.

20. Gupta K, Hooton TM, Wobbe CL, Stamm WE (1999): The prevalence of antimicrobial resistance among uropathogens causing acute uncomplicated cystitis in young women.Int $\mathrm{J}$ Antimicrob Agents., 11(3-4):305-8. 